Rakenteiden Mekaniikka (Journal of Structural Mechanics)

Vol. 50, No 3, 2017, pp. $372-375$

https://rakenteidenmekaniikka.journal.fi/index

https://doi.org/10.23998/rm.65180

(c) The author(s) 2017.

Open access under CC BY-SA 4.0 license.

\title{
Preliminary results on the effect of plastic deformation on magnetic properties
}

\author{
Deepak Singh, Kati Mökkönen, Jarmo Poutala, Paavo Rasilo ${ }^{1}$, Anouar Belahcen, and \\ Reijo Kouhia
}

Summary. In this paper some preliminary results on the effect of plastic deformation on magnetic properties of an electric steel are presented. Both experimental results and modelling aspects are discussed.

Key words: magnetic properties, electric steel, plastic deformation

Received 11 June 2017. Accepted 21 August 201\%. Published online 21 August $201 \%$.

\section{Introduction}

Stresses are known to influence significantly on the magnetic properties of electric steels. The laminated core in electrical motors is fabricated from electric steel sheets which are punched, compressed and welded together. Punching the electric steel sheets produces significant plastic deformations near the edges. Although these plastic regions are small in volume they can have a significant effect on the core losses in electrical machines [1].

In this study, an experimental investigation of the magnetic properties of electric steel sheet of grade M400-50A have been performed. The magnetic flux density is determined as a function of the magnetic field strength for pre-strained samples loaded to permanent plastic deformation of 5,10 and $15 \%$. Also some issues related to the magneto-mechanical modelling are discussed.

\section{Experiments}

The MTS 810 loading frame, control system and transducers were used to get permanent plastic strains for rolling and transversal oriented standardized samples. The MTS 810 tensile machine and all transducers are calibrated annually on a regular basis by official site. The drawing speed was $10 \mathrm{~mm} / \mathrm{min}$. The used extensometer has a range of $\pm 10 \%$ with the gap of $25 \mathrm{~mm}$ between fixing knives. The extensometer was attached in the middle of the samples by two rubber bands. The range of the force transducer of the system is $\pm 100 \mathrm{kN}$. The piston was position controlled during the tests. The data acquisition took

\footnotetext{
${ }^{1}$ Corresponding author. paavo.rasilo@tut.fi
} 

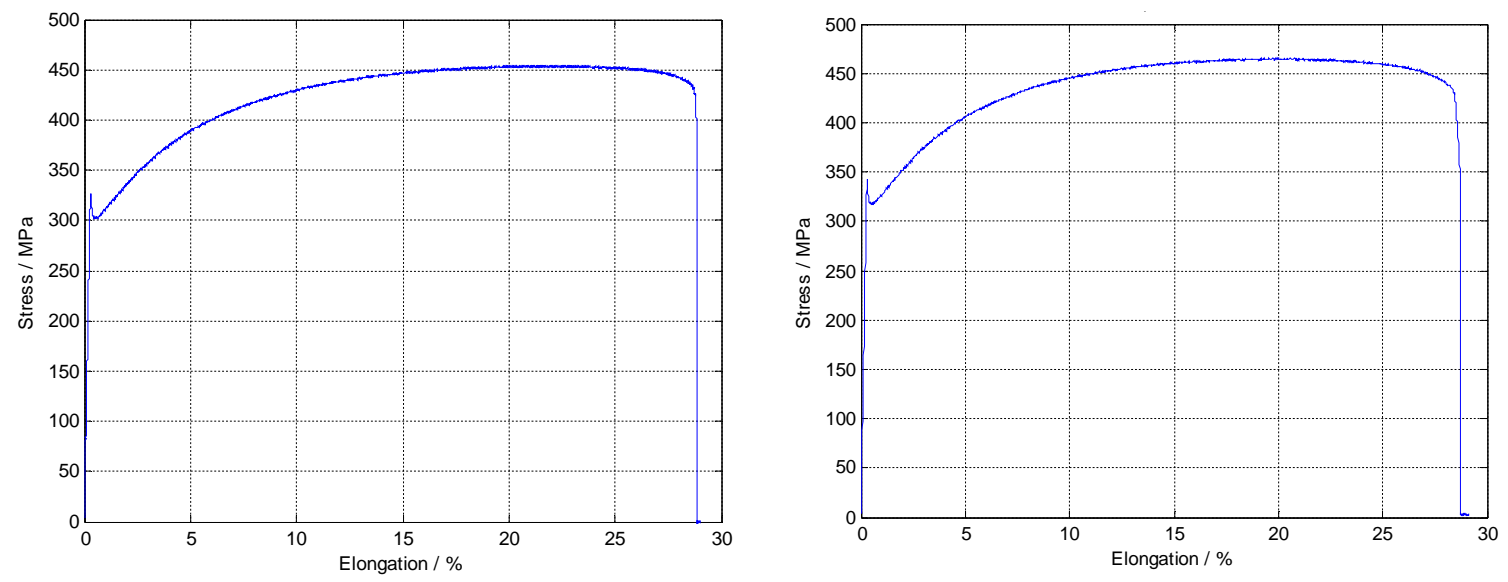

Figure 1. Stress-strain behaviour in the rolling direction (LHS) and in the transverse direction (RHS).

place at the rate of $20 \mathrm{~Hz}$. The samples were pulled by hydraulic grips. The specimen were placed in position between the grips under the control of force to avoid any damage during the first preparations before pulling. The extensometer was used in tension and to measure strains from the undeformed state to the permanent plastic deformations of 5 and $10 \%$. Strains over $10 \%$ were measured only by the frame displacement transducer which was programmed to give directly also strains in addition to displacement data. When pulling the samples to those higher deformations the extensometer was taken away first at the level of $10 \%$ halting the pull momentarily and then continued to $15 \%$. All quantities were measured simultaneously and analyzed after the test. All measurements were verified also by the slide caliper after every tensile test with the help of two drawn lines in the middle of the sample. The distance between these drawn lines was $20 \mathrm{~mm}$.

The stress-strain relationship is determined in both rolling and transverse directions. It seems that there is no significant plastic anisotropy. The lower yield stress is in the range $300-320 \mathrm{MPa}$ and the ultimate tensile strength between $450-470 \mathrm{MPa}$, see Fig. 1 . It can also be seen from these stress and strain graphs that the localization of the plastic deformation occurs after strain level of approximately $20 \%$.

The unidirectional magnetic measurements were done on the plastically deformed single sheet testers (SST) samples. Tunneling magneto-resistance (TMR) sensors of dimension $6 \times 5 \times 1.5 \mathrm{~mm}$ arranged in a $2 \times 2$ grid on a printed circuit board of $20 \times 20 \mathrm{~mm}$ were used to measure the surface magnetic field strength. Similarly, a coil wound around the SST sample was used to measure the average magnetic flux density. A programmable power source (AMETEK CSW 5550VA) and a data acquisition system (DAQ-NI USB$6251 \mathrm{BNC}$ ) with analog output were used in conjunction with a PC to control the magnitude and the waveform of the supply voltage so as to produce a sinusoidal induction in the SST sample. The feedback control of the supply voltage was programmed using the MATLAB/DAQ toolbox. Low-noise/high-gain signal amplifiers were also used to amplify the signal obtained from the search coils. In addition to that, a high sampling rate DAQ system (DEWETRON) controlled by PC/DEWEsoft was used to retrieve the measured signals for the field strength and the flux density. The sample was magnetized using two vertical cores and the excitation coils with a total of 1000 turns. Within the limitation of the power amplifier's operation, the total turns of the excitation coils were sufficient for wide range of induction amplitudes $(0.2 \mathrm{~T}$ to $1.5 \mathrm{~T})$ and frequencies $(6 \mathrm{~Hz}$ to $150 \mathrm{~Hz})$.

Single-valued magnetization curves are shown in Fig. 2 for both rolling and transverse directions. Although the mechanical properties are almost isotropic, the magnetic 


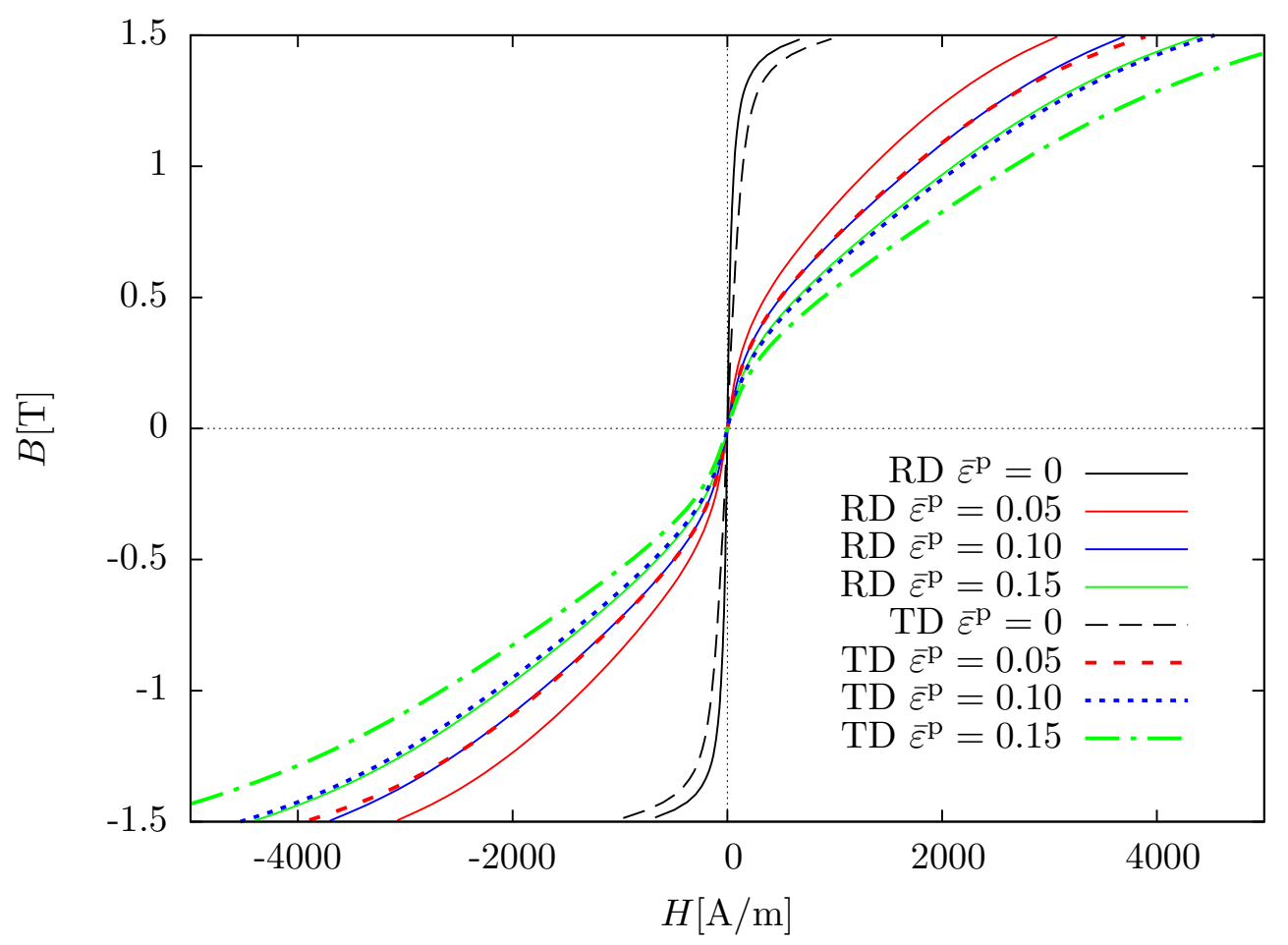

Figure 2. Single valued magnetic flux density curve as a function of magnetic field strength with different values of plastic deformation. Lines denoting rolling direction results are drawn by solid lines and tranverse direction results by dashed lines.

properties show clear directionality which should be taken into account in the modelling.

\section{Modelling aspects}

Extension to the coupled magnetomechanical model described in [2] is needed. In this model the reversible behaviour is described by a special choice of the specific Helmholtz free energy, expressed in terms of the invariants forming the integrity basis of a scalar function depending on a symmetric second order tensor and a vector, i.e. the strain tensor $\boldsymbol{\varepsilon}$ and the magnetic flux density $\boldsymbol{B}[3,4]$. Restricting to small strains, the Helmholtz free energy is formulated in terms of elastic strain tensor $\varepsilon^{\mathrm{e}}=\varepsilon-\varepsilon^{\mathrm{p}}$, magnetic flux density $\boldsymbol{B}$ and an internal variable, which in the case of isotropically hardening von Mises plastic model is the equivalent plastic strain, defined as

$$
\bar{\varepsilon}^{\mathrm{p}}=\int \dot{\bar{\varepsilon}}^{\mathrm{p}} \mathrm{d} t, \quad \dot{\bar{\varepsilon}}^{\mathrm{p}}=\sqrt{\frac{2}{3} \dot{\varepsilon}_{i j}^{\mathrm{p}} \dot{\varepsilon}_{i j}^{\mathrm{p}}}
$$

For associative plastic flow the plastic strain rate is obtained from the plastic potential as

$$
\dot{\boldsymbol{\varepsilon}}^{\mathrm{p}}=\lambda \frac{\partial f}{\partial \boldsymbol{\sigma}},
$$

where $f$ is the yield function and $\lambda$ is the plastic multiplier to be determined from the consistency condition. Stresses $\boldsymbol{\sigma}$ and magnetization $\boldsymbol{M}$ are obtained from the specific Helmholtz free energy $\psi=\psi\left(\varepsilon^{\mathrm{e}}, \boldsymbol{B}, \bar{\varepsilon}^{\mathrm{p}}\right)$ as

$$
\boldsymbol{\sigma}=\rho \frac{\partial \psi}{\partial \boldsymbol{\varepsilon}^{\mathrm{e}}}, \quad \boldsymbol{M}=-\rho \frac{\partial \psi}{\partial \boldsymbol{B}},
$$

where $\rho$ is the density. 


\section{Concluding remarks}

It appears that a rather small permanent plastic deformation $\left(\bar{\varepsilon}^{\mathrm{p}}<5 \%\right)$ has a pronounced effect on the magnetic properties. Further experiments will be conducted to get more detailed information in this range. However, for proper numerical simulations a finite strain plasticity model should be used to get reliable results in the analyses.

\section{Acknowledgements}

The research leading to these results has received funding from the European Research Council under the European Union's Seventh Framework Programme (FP7/2007-2013) / ERC grant agreement $\mathrm{n}^{\circ} 339380$, from a strategic grant of Tampere University of Technology, from the Challenge Finland project "Clean Energy from Environment with Energy Harvesting" funded by Tekes - the Finnish Funding Agency for Innovation, and from the Academy of Finland grants 274593 and 304112.

\section{References}

[1] D. Singh. Effect of stress on magnetic properties of electrical steel sheet and core losses in electrical machines. PhD-thesis, Aalto University, 2016.

[2] K. Fonteyn, A. Belahcen, R. Kouhia, P. Rasilo, A. Arkkio. FEM for directly coupled magneto-mechanical phenomena in electrical machines. IEEE Transactions on Magnetics, 46(8):2923-2926, 2010. https://doi.org/10.1109/TMAG.2010.2044148.

[3] A. Kovetz. Electromagnetic Theory. Oxford University Press, 2000.

[4] A.J.M. Spencer. Theory of invariants. In A.C. Eringen, editor, Continuum Physics, volume 1, pages 239-353. Academic Press, 1971. https://doi .org/10.1016/ B978-0-12-240801-4.50008-X.

Deepak Singh, Paavo Rasilo

Tampere University of Technology, Electrical Energy Engineering, P.O. Box 692, FI-33101 Tampere, Finland

deepak.singh@tut.fi, paavo.rasilo@tut.fi

Anouar Belahcen

Aalto University, Electrical Engineering, P.O. Box 13000, FI-00076 Aalto, Finland

anouar.belahcen@aalto.fi

Kati Mökkönen

Tampere University of Technology, Materials Science, P.O. Box 589, FI-33101 Tampere, Finland

kati.mokkonen@tut.fi

Jarmo Poutala, Reijo Kouhia

Tampere University of Technology, Civil Engineering, P.O. Box 600, FI-33101 Tampere, Finland

jarmo.poutala@tut.fi, reijo.kouhia@tut.fi 